\title{
86. A Characterization of Hyperplane Cuts of Smooth Complete Intersections
}

\author{
By Shihoko IsHII \\ Department of Mathematics, Tokyo Metropolitan University \\ (Communicated by Kunihiko KodaIra, M. J. A., Sept. 13, 1982)
}

In this note, we will prove the following

Theorem. Let $M \subset \boldsymbol{P}^{N+1}$ be a smooth complete intersection. We assume for simplicity that $M$ is non-degenerate, i.e., $M$ is not contained in any linear subspace of $\boldsymbol{P}^{N+1}$. Then any hyperplane section $X$ of $M$ has the following two properties:

(A) $X$ has only finitely many singular points;

(B) The Jacobian matrix $J$ of $X \subset \boldsymbol{P}^{N}$ has rank $r-1$ at any singular point of $X$.

Conversely, if $X \subset \boldsymbol{P}^{N}$ is a non-degenerate complete intersection having the properties (A) and (B), then there exists a smooth complete intersection $M \subset \boldsymbol{P}^{N+1}$ such that $X$ is a hyperplane section of $M$.

Remark. The property (A) implies that $X$ is reduced if $\operatorname{dim} M \geq 2$ and irreducible if $\operatorname{dim} M \geq 3$. Moreover, (A) is a partial refinement of the following

Zak's Theorem (see [1]). Let $M \subset \boldsymbol{P}^{N+1}$ be an irreducible smooth non-degenerate subvariety of codimension $r$ and $X$ an arbitrary hyperplane section of $M$. Then the dimension of the singular locus of $X$ is less than $r$.

In [1], the property (A) is shown by using a suitable incidence correspondence. Our proof is more direct and elementary.

Throughout this note, we fix an algebraically closed field $k$ of any characteristic and assume that all varieties are defined over $k$.

Proof of Theorem. For brevity, we introduce a symbol $V\left(F_{1}, \ldots\right.$, $F_{r}$ ) which stands for the projective variety defined by the homogeneous polynomials $F_{1}, \cdots, F_{r}$. For a given smooth complete intersection $M \subset \boldsymbol{P}^{N+1}$, we write $M=V\left(\tilde{F}_{1}, \ldots, \tilde{F}_{r}\right)$, where $\tilde{F}_{i}$ is a homogeneous polynomial of degree $d_{i} \geq 2$ in $Z_{0}, Z_{1}, \ldots, Z_{N+1}$. By a suitable linear transformation of the coordinates, we may assume that

$$
X=M \cap\left\{Z_{N+1}=0\right\}=V\left(\tilde{F}_{1}, \ldots, \tilde{F}_{r}, Z_{N+1}\right) .
$$

Putting $F_{i}\left(Z_{0}, \cdots, Z_{N}\right)=\tilde{F}_{i}\left(Z_{0}, \cdots, Z_{N}, 0\right)$, we write

$$
\tilde{F}_{i}\left(Z_{0}, \ldots, Z_{N+1}\right)=F_{i}\left(Z_{0}, \cdots, Z_{N}\right)+Z_{N+1} G_{i}\left(Z_{0}, \ldots, Z_{N+1}\right) \text {. }
$$

Denote by $\widetilde{J}(p)$ and $J(p)$ the Jacobian matrices of the defining equations $\left\{\tilde{F}_{1}, \ldots, \tilde{F}_{r}\right\}$ and $\left\{F_{1}, \ldots, F_{r}\right\}$ at $p \in X$, respectively. Then, since $Z_{N+1}=0$ on $X$, we have 


$$
\tilde{J}(p)=\left(\begin{array}{c}
J(p) \\
G_{1}(p), \cdots, G_{r}(p)
\end{array}\right) .
$$

Since $M$ is smooth, $\operatorname{rank} \tilde{J}(p)=r$. So we have rank $J(p) \geq r-1$. This implies (B).

Let $S$ be an irreducible component of the singular locus of $X$. We assume that $S$ is of maximal dimension. Noting that rank $J(p)$ $=r-1$ at $p \in S \subset X$, we choose a non-zero vector $\left(a_{1}(p), \cdots, a_{r}(p)\right)$ for each $p \in S$ such that

$$
\sum_{i=1}^{r} a_{i}(p) \frac{\partial F_{i}}{\partial Z_{j}}(p)=0 \quad(j=0, \cdots, N) .
$$

Then the vector $f(p)=\left(a_{1}(p) G_{1}(p), \cdots, a_{r}(p) G_{r}(p)\right)$ determines a point in $\boldsymbol{P}^{r-1}$, which does not depend on the vector $\left(a_{1}(p), \ldots, a_{r}(p)\right)$. In fact, if $a_{1}(p) G_{1}(p)=\cdots=a_{r}(p) G_{r}(p)=0$, then $\sum a_{i}(p)\left(\partial \tilde{F}_{i} / \partial Z_{j}\right)(p)=0$ and $\tilde{J}(p)$ would have rank $\leq r-1$. Thus, $f: S \rightarrow P^{r-1}$ is a morphism. Assume that $f$ is not a constant map. Then $f(S) \cap\left\{Y_{1}+\cdots+Y_{r}=0\right\} \neq \phi$, where $\left\{Y_{1}, \ldots, Y_{r}\right\}$ are the homogeneous coordinates of $\boldsymbol{P}^{r-1}$. This implies that

$$
\sum a_{i}(p) G_{r}(p)=\sum a_{i}(p) \frac{\partial F_{i}}{\partial Z_{j}}(p)=0 \quad(j=0,1, \cdots, N)
$$

for some point $p \in S$ and $M$ would not be smooth at $p$. Hence $f$ must be a constant map, and $a_{i}(p) G_{i}(p)$ never vanish on $S$ for some $i$. This is possible only when $\operatorname{dim} S=0$ since $\operatorname{deg} G_{i}=d_{i}-1 \geq 1$.

Now, let $X$ be a complete intersection $V\left(F_{1}, \ldots, F_{r}\right)$ in $P^{N}$ ( $d_{i}=\operatorname{deg} F_{i} \geq 2$ ), and assume that $X$ has the above properties (A) and (B). Let $S$ be the finite singular locus of $X$ and let $G_{i}^{(k)}\left(Z_{0}, \ldots, Z_{N}\right)$ be a general homogeneous polynomial of degree $d_{i}-k$. Set $\tilde{F}_{i}\left(Z_{0}, \cdots\right.$, $\left.Z_{N+1}\right)=F_{i}\left(Z_{0}, \ldots, Z_{N}\right)+\sum_{k=1}^{d_{i}} Z_{N+1}^{k} G_{i}^{(k)}\left(Z_{0}, \ldots, Z_{N}\right)$. Then the Jacobian matrix $\tilde{J}(p)$ of $M=V\left(F_{1}, \cdots, F_{r}\right) \subset \boldsymbol{P}^{N+1}$ at $p \in X$ is $\left(\begin{array}{c}J(p) \\ G_{1}^{(1)}(p), \cdots, G_{r}^{(1)}(p)\end{array}\right)$. Since $S$ is finite and $G_{i}^{(1)}$ is general, $\operatorname{rank} \tilde{J}(p)$ is equal to $r$ at $p \in S$. Hence $\tilde{J}$ has rank $r$ everywhere on $X=M \cap V\left(Z_{N+1}\right)$. On the other hand, $M \backslash X$ is non-singular in virtue of the following

Lemma. Let $f_{1}, \cdots, f_{r}$ be polynomials in $x_{1}, \cdots, x_{n}(1 \leq r \leq n)$. Then the affine variety in $\boldsymbol{A}^{n}$ defined by

$$
f_{i}+\sum_{j=1}^{n} a_{i j} x_{j}+a_{i n+1} \quad(i=1, \cdots, r)
$$

is non-singular for general coefficients $\left(a_{i j}\right) \in k^{r(n+1)}$.

Remark. The above theorem fails for a general smooth submanifold $M \subset \boldsymbol{P}^{N+1}$. Indeed, a hypersurface section of $M$ satisfies neither (A) nor (B) in general. Therefore, the Veronese embedding of $M$ gives a counterexample. 


\section{Reference}

[1] W. Fulton and R. Lazarsfeld: Connectivity and its applications in algebraic geometry. Lect. Note in Math., vol. 862, Springer-Verlag, pp. 26$92(1980)$. 